Man and Nature

L'homme et la nature

\title{
Diderot entre les « Têtes froides " et les « Enthousiastes "
}

\section{Roland Mortier}

Volume 6, 1987

URI : https://id.erudit.org/iderudit/1011866ar

DOI : https://doi.org/10.7202/1011866ar

Aller au sommaire du numéro

Éditeur(s)

Canadian Society for Eighteenth-Century Studies / Société canadienne d'étude du dix-huitième siècle

ISSN

0824-3298 (imprimé)

1927-8810 (numérique)

Découvrir la revue

Citer cet article

Mortier, R. (1987). Diderot entre les « Têtes froides " et les « Enthousiastes ". Man and Nature / L'homme et la nature, 6, 1-19.

https://doi.org/10.7202/1011866ar

Copyright (C Canadian Society for Eighteenth-Century Studies / Sociéte canadienne d'étude du dix-huitième siècle, 1987
Ce document est protégé par la loi sur le droit d'auteur. L'utilisation des services d'Érudit (y compris la reproduction) est assujettie à sa politique d'utilisation que vous pouvez consulter en ligne.

https://apropos.erudit.org/fr/usagers/politique-dutilisation/ 


\section{DIDEROT ENTRE LES “TÊTES FROIDES" ET LES “ENTHOUSIASTES"}

Sur bien des points importants, qui touchent à l'esthétique, à la morale et à la politique, la pensée de Diderot ne se prête pas à une définition simple et tranchée. Oscillante de nature, toujours sollicitée par d'autres voix, elle se refuse aux tentatives de réduction unilatérale, comme le penseur lui-même se refusait à toute forme d'adhésion inconditionnelle. Très tôt, on l'a accusé de versatilité, et lui-même y a prêté la main en se comparant un jour au coq sans cesse en mouvement sur le clocher de Langres. De là à tenir sa pensée pour inconsistante, ou volontairement paradoxale, donc décevante (dans tous les sens du terme), il n'y avait qu'un pas, qu'une certaine critique n'a pas hésité à franchir.

En réalité, cette pensée est très loin d'être négative, ou mue par le seul désir de surprendre. Elle est, au contraire, sensible à la complexité de la vie et des êtres, consciente aussi de ses propres insuffisances. Elle varie comme la personnalité de l'homme qu'elle habite: “J'avais en un jour cent physionomies diverses", parce qu'elle participe de la mobilité du vivant et qu'elle ne confond pas fidélité avec fermeture ou dogmatisme, mais avec ouverture et diversité. Toute l'oeuvre de Diderot procède de cette recherche d'une vérité sans cesse fuyante, parce qu'irréductible aux abstractions et aux systèmes. Faut-il y voir une faiblesse ou une force? Ce serait mal poser la question. Pour lui, chaque prise de position se réfère à un donné particulier, passé ou présent, par rapport auquel il doit se situer. Toute systématisation en- 
treprise au mépris du réel, toute généralisation proférée au détriment du particulier et de l'instant lui sont insupportables; il n'y voit qu'excès de l'intelligence raisonneuse, outrance de notre orgueil intellectuel. Sa parenté d'esprit avec Montaigne est évidente sur ce point. Il cherche la vérité dans la nuance, dans le subtil accord avec une réalité irréductible à nos catégories préfabriquées. Pensée questionneuse donc, et qui prend plus de plaisir à créer des nuages qu'à les dissiper, comme Lessing l'avait si justement remarqué dès la lecture de la Lettre sur les sourds et muets.

Pourquoi, dès lors, s'étonnerait-on de ses variations à propos de l'enthousiasme ou, inversement, de son panégyrique des esprits "froids et tranquilles" dans le Paradoxe sur le Comédien?

Sans doute faut-il, dans une juste appréciation de ces flottements, tenir compte de la chronologie et de l'évolution qu'elle suppose dans sa pensée. Mais l'alternance, dans le cas présent, ne se réduit pas à un avant et à un après. Elle touche à un questionnement plus profond, à une oscillation qui tient à la nature même de la pensée de Diderot, solicitée à la fois, et contradictoirement, par l'enthousiasme et par la lucidité, le tout étant affaire de dosage, de circonstance et de sujet.

Encore convient-il de s'entendre au préalable sur le (ou les) sens qu'ont les mots enthousiasme et enthousiaste au début du XVIIIe siècle. ${ }^{1}$ L'un d'eux est résolument négatif: il désigne un délire inspiré, une exaltation folle, un fanatisme aveugle, un dévoiement de la sensibilité, toutes manifestations tenues alors pour morbides ou pathologiques. Cette interprétation est d'origine cartésienne ou libertine; dans d'autres cas, elle découle de la dégradation subie par le mot anglais correspondant, à la suite des extravagances et des aberrations qui s'étaient manifestées dans le milieu prophétique des "camisards" réfugiés et qui trouvaient leur corollaire français dans les phénomènes paranormaux observés sur la tombe du diacre janséniste Pâris au cimetière de SaintMédard. Parmi les exemples relevés par Fritz Schalk, on retiendra quelques expressions très significatives de ce rejet:

- Saint-Evremond: "ces enthousiasmes de religion qui rendent la vie odieuse à tant de fanatiques"

- Malebranche: "ce feu, ces emportements, ces enthousiasmes sur de petits sujets marquent sensiblement le dérèglement de son imagination" (il s'agit de Tertullien)

- Marivaux: "les discours d'enthousiaste et d'inspiré que Cromwell tenait souvent dans l'armée" 
- Burton (Anatomy of Melancholy): "out of presumption of their holiness and good gifts, become prophets, enthusiasts, and what not?" (contre la pathologie des sectes).

Shaftesbury va dans le même sens lorsqu'il publie en 1708 A letter concerning enthusiasm, tout comme le fera David Hume, en 1758, dans un Essay on superstition and enthusiasm. Richard Hurd, en sa qualité d'évêque de Worcester, dénonce en 1752, dans un sermon aussitôt publié, The mischiefs of enthusiasm and bigotry.

En Allemagne, Reimarus accuse tous les "inspirés" de pervertir la religion naturelle. Aussi met-il dans le même sac théosophes, quakers, piétistes, chiliastes, boehmistes, fanatiques, méthodistes, Herrnhuter, et autres mystiques, tous possédés par "un esprit fanatique et enthousiaste", et forme-t-il le voeu qu'un jour quelqu'un écrive l'histoire de cette aberration (Schützschrift für die vernünftigen Verehrer Gottes, éd. 1972, I, 164 ss.). Le Dictionnaire de Trévoux, organe des Jésuites, considère "enthousiaste" comme un synonyme de "fanatique, visionnaire" et cite en exemple les Quakers et les anabaptistes dans une notice d'un ton très hostile (éd. de 1771).

Les matérialistes français du groupe holbachique emploient ces mêmes termes dans un sens très critique, qui se confond pour eux avec l'aliénation religieuse et le fanatisme aveugle. La Révolution, puis Madame de Staël, et enfin les romantiques, réhabiliteront entièrement la notion d'enthousiasme, dont ils feront le principe même de toute élévation spirituelle. ${ }^{2}$

Le jeune Diderot, fortement marqué par l'influence de Shaftesbury, oscille entre ce sens négatif et la conception néo-platonicienne de l'enthousiasme conçu comme un don divin, un état supérieur de l'esprit, héritage de la Renaissance italienne (Giordano Bruno) et française (Ronsard, Pontus de Tyard, Agrippa d'Aubigné). Ce flottement est très net dans les Pensées philosophiques (1746), mais il résulte au moins autant d'une confusion sémantique que d'une absence de rigueur théorique. Lorsqu'il écrit, dans la pensée XXXIX: "Le vrai martyr attend la mort; l'enthousiaste y court", il reprend le point de vue de Shaftesbury et des tenants de la religion naturelle et raisonnable. On y retrouve l'écho de la dédicace $A$ mon frère de l'Essai sur le mérite et la vertu où il considérait Didier-Pierre comme un "ennemi de l'enthousiasme et de la bigoterie" (éd. Hermann, I, 291). La pensée XXVIII est plus complexe et va plus loin. Elle s'inscrit dans une série où il fait l'éloge du scepticisme et qui traite moins de religion que d'épistémologie. 
“Les esprits bouillants, les imaginations ardentes ne s'accommodent pas de l'indolence du sceptique. Ils aiment mieux hasarder un choix que de n'en faire aucun, se tromper que de vivre incertains ... Ils assurent tout, bien qu'ils n'aient rien soigneusement examiné: ils ne doutent de rien, parce qu'ils n'en ont ni la patience, ni le courage. Sujets à des lueurs qui les décident, si par hasard ils rencontrent la vérité, ce n'est point à tâtons, c'est brusquement, et comme par révélation. Ils sont, entre les dogmatiques, ce qu'on appelle les illuminés chez le peuple dévot. J'ai vu des individus de cette espèce inquiète qui ne concevaient pas comment on pouvait allier la tranquillité d'esprit avec l'indécision".

Pour le sceptique, c'est-à-dire pour le jeune Diderot de 1746: "Toute ma vie j'ignorerai, sans chagrin, ce qu'il m'est impossible de savoir. Pourquoi regretterais-je des connaissances que je n'ai pu me procurer, et qui, sans doute, ne me sont pas fort nécessaires, puisque j'en suis privé?". Diderot ne se satisfera pas très longtemps de ces limites imposées à notre besoin de connaître. Reste en tout cas qu'il parle des "imaginations ardentes" sans malveillance aucune et qu'il constate objectivement qu'il leur arrive de "rencontrer la vérité comme par révélation". S'il s'oppose à cette famille d'esprits, ce n'est ni par paresse intellectuelle, ni par incuriosité, mais parce qu'il constate qu'ils "aiment mieux de demeurer accorchés à une branche que de s'abandonner au torrent". La formule a de quoi surprendre le lecteur, qui s'attendrait plutôt à voir le sceptique dans cette position d'attente. Certes, la pensée suivante éclairera un peu mieux l'idée que Diderot se fait, à ce moment, de la recherche de la vérité, mais ce tâtonnement et ces faux pas se concilient malgré tout assez mal avec l'image du torrent auquel s'abandonne l'esprit. Il semble que Diderot, en 1746, soit encore sollicité simultanément par la chaleur des passions, justifiées dans les pensées I à $V,{ }^{3}$ et par le paisible détachement du sceptique, esprit froid $s^{\prime}$ il en est. ${ }^{4}$

Son point de vue a, en tout cas, considérablement évolué lorsqu'il donne, en 1753, les Pensées sur l'interprétation de la nature. L'expérience déjà acquise dans l'Encyclopédie, la maturation de sa réflexion personnelle, le contact avec les expérimentateurs, tout cela a contribué à enrichir et à nuancer sa conception de la recherche et de l'attitude intellectuelle qu'elle suppose. Il ne croit plus à la recherche erratique et tâtonnante, livrée au hasard et progressant par à-coups.

Il a compris que toute recherche est d'abord un questionnement, qu'elle exige une longue pratique et s'inscrit dans une visée presque programmatique, qu'elle s'accommode très mal de "l'indolence du sceptique". Diderot va s'efforcer de récupérer une faculté authentiquement 
divinatoire en excluant les connotations religieuses et mystiques qui lui sont trop souvent associées dans la pratique du langage.

C'est évidemment la pratique des ateliers et des laboratoires qui l'amène à faire l'éloge des "manouvriers d'expériences", opposés aux philosophes trop théoriciens dans la pensée XVI. La pensée XXX revient plus longuement sur la nature à la fois très pratique et assez mystérieuse de leur don.

“La grande habitude de faire des expériences donne aux manouvriers d'opérations les plus grossiers un pressentiment qui a le caractère de l'inspiration. Il ne tiendrait qu'à eux de s'y tromper comme Socrate, et de l'appeler un démon familier ... Ils ont vu si souvent et de si près la nature dans ses opérations, qu'ils devinent avec assez de précision le cours qu'elle pourra prendre dans le cas où il leur prend envie de la provoquer par les essais les plus bizarres. Aussi le service le plus important qu'ils aient à rendre à ceux qu'ils initient à la philosophie expérimentale, c'est bien moins de les instruire du procédé et du résultat, que de faire passer en eux cet esprit de divination par lequel on subodore, pour ainsi dire, des procédés inconnus, des expériences nouvelles, des résultats ignorés".

Revenant sur ce problème qui semble vraiment l'obséder, il explique cette étrange capacité divinatoire par la pratique constante de l'analogie, démarche cependant moins scientifique que poétique (comme il le dira lui-même dans Le rêve de d'Alembert, où la même thématique reparaît. ${ }^{5}$ La genèse de leur faculté divinatoire, si les "manouvriers d'expériences" voulaient ou pouvaient la communiquer, "serait une histoire fidèle de toutes les extravagances apparentes qui leur ont passé par la tête". Et il ajoute (p. XXXI): "Je dis extravagances; car quel autre nom donner à cet enchaînement de conjectures fondées sur des oppositions ou des ressemblances si éloignées, si imperceptibles, que les rêves d'un malade ne paraissent ni plus bizarres, ni plus décousus?" Il y revient avec insistance dans la Pensée XXXII (Premières conjectures): "C'est cette habitude de déraison que possèdent dans un degré surprenant ceux qui ont acquis ou qui tiennent de la nature le génie de la physique expérimentale; c'est à ces sortes de rêves qu'on doit plusieurs découvertes. Voilà l'espèce de divination qu'il faut apprendre aux élèves, si toutefois cela s'apprend".

Remarquons simplement que cet "esprit de divination" n'a aucun rapport avec une mystique ou une source religieuse. S'il tient du rêve et de la déraison, c'est qu'il fait appel à l'imagination, à la fantaisie, à la capacité d'associer les idées les plus diverses, comme le faisait Socrate, "dans une combinaison prompte et juste". 
Il en viendra, sur sa lancée, à opposer au simple "observateur de la nature" celui qu'il appelle l'interprète de la nature, seul capable de s'élever, au-delà du multiple, jusqu'à "l'essence même de l'ordre" ( $p$. LVI). Par ce biais, Diderot récupère une faculté généralement rapportée à une présence divine ou démonique, quand on ne la confinait pas dans l'ordre poétique.

Dans les mêmes Pensées surgit soudain, au terme de "conjectures sur l'imitation de la nature" (XXXVII), un rejet presque passionnel de l'admiration "froide et stérile" qu'inspire à certains le "goût antique pour les grandes entreprises". L'association froideur-stérilité explicite assez clairement l'aversion que devaient lui inspirer, à cette date, les "esprits froids". Sans doute les associe-t-il à ces esprits systématiques, qu'il appelle ailleurs "méthodistes", et dont il dit qu'au lieu de réformer leurs notions sur les êtres, ils prennent à tâche de modeler les êtres sur leurs notions (XLVIII).

L'Encyclopédie nous permet d'élargir l'enquête, à la fois dans les sujets et dans le temps. L'article Divination renvoie certes à Prophétie et à Enthousiasme, mais se maintient au plan religieux et historique. Eclectisme aborde le problème de front: "l'enthousiasme divin" y est dépeint comme une étrange aliénation.

“Celui qui l'éprouve est privé de l'usage commun de ses sens ... son action est extraordinaire; il ne se possède plus; il ne pense plus et ne parle plus par lui-même; il ne sent point l'action du feu, ou il n'en est point offensé; il ne voit ni ne redoute la hache levée sur sa tête; il est transporté dans des lieux inaccessibles, il marche à travers la flamme; ' il se promène sur les eaux, etc." (éd. Hermann, VII, 107-108)

Cette forme de possession intérieure ne peut que lui sembler suspecte. L'enthousiaste, "ministre d'un dieu qui l'obsède", est un être qui ne raisonne plus et qui se sent "élevé au-dessus de la nature commune". ${ }^{7}$ Il peut être un martyr, mais aussi un redoutable fanatique et un impitoyable persécuteur. Diderot lui préfère, personnellement, les philosophes dits "éclectiques", c'est-à-dire indépendants (puisque le penseur "éclectique" est "un homme qui recueille et qui crible").

L'éclectisme a permis à la libre pensée de survivre à l'intolérance des premiers chrétiens tout en maintenant la paix sociale. Ici se situe le premier éloge des "esprits froids":

“Le soulèvement des prêtres, du peuple, et des philosophes, aurait été général, sans un petit nombre d'hommes froids, comme il s'en trouve toujours dans les sociétés, qui demeurent longtemps spectateurs indifférents, qui écou- 
tent, qui pèsent, qui n'appartiennent à aucun parti, et qui finissent par se faire un système conciliateur, auquel ils se flattent que le grand nombre reviendra. Telle fut à peu près l'origine de l'éclectisme". (éd. Hermann, VII, 39).

L'article Consolation évoque le fanatisme des Albigeois et leur indifférence à la mort, avant de conclure: "Exemple frappant de ce que peuvent l'enthousiasme et la superstition, lorsqu'ils se sont une fois fortement emparés des esprits" (VI, 490). Il s'agit, bien entendu, du sens religieux du terme. On le retrouve, un peu dans le même sens, à l'article Impassible, Impassibilité (VII, 505): “Une mère qui verrait son enfant en danger courrait à son secours les pieds nus à travers des charbons ardents, sans en ressentir de douleur. L'enthousiasme et le fanatisme peuvent élever l'âme au-dessus des plus affreux tourments. Voyez dans le livre de la Cité de Dieu, l'histoire du prêtre de Calame. Cet homme s'aliénait à son gré, et se rendait impassible même par l'action du feu".

L'article Fureur (VII, 313-314) apporte quelques utiles précisions qui permettent de nuancer les degrés d'enthousiasme dans le domaine artistique. A première vue, à son degré extrême, la fureur poétique devrait coïncider avec les types de discours où les règles sont les moins contraignantes, et particulièrement avec la conversation. Or c'est l'inverse qui se vérifie. "Il est plus essentiel au musicien d'être enthousiaste qu'au poète, au poète qu'au peintre, au peintre qu'à l'orateur, et à l'orateur qu'à l'homme qui converse. L'homme qui converse ne doit pas être froid, mais il doit être tranquille".

Mais l'article le plus important pour notre propos est très certainement celui sur les Théosophes (VIII, 365-393), dont l'intérêt a été souligné par Jean Fabre dès $1960 .{ }^{8} \mathrm{Il}$ commence par affirmer, à la suite de Brucker, que ces philosophes "regardaient en pitié la raison humaine; ils n'avaient nulle confiance dans sa lueur ténébreuse et trompeuse; ils se prétendirent éclairés par un principe intérieur, surnaturel et divin, qui brillait en eux et s'y éteignait par intervalles, qui les élevait aux connaissances les plus sublimes lorsqu'il agissait, ou qui les laissait tomber dans l'état d'imbécillité naturelle lorsqu'il cessait d'agir; qui s'emparait violemment de leur imagination, qui les agitait, qu'ils ne maîtrisaient pas, dont ils étaient maîtrisés, et qui les conduisait aux découvertes les plus importantes et les plus cachées sur Dieu et sur la nature." On pourrait supposer que leur irrationalisme de principe, leur attitude presque mystique à l'égard de la connaissance, leur croyance en une lumière intérieure d'origine divine, susciteraient l'ironie, voire l'hostilité de Diderot, matérialiste décidé et défenseur des valeurs rationnelles. Loin de là: notre encyclopédiste s'empresse de prendre ses distances par rapport aux critiques de la théosophie. Ils ont, dit-il, 
"passé pour des fous auprès de ces hommes tranquilles et froids dont l'âme pesante ou rassise n'est susceptible ni d'émotion ni d'enthousiasme, ni de ces transports dans lesquels l'homme ne voit point, ne sent point, ne juge point, ne parle point, comme dans son état habituel." Ce sont les mêmes qui ont tourné en dérision ou taxé de friponnerie le démon de Socrate.

Et Diderot d'intervenir personnellement dans le débat par une longue digression qui s'ouvre sur cette question: "Me sera-t-il permis de dire un mot en faveur du démon de Socrate et de celui des théosophes?" Car il se sent proche de ces hommes, doués comme lui "d'une grande sensibilité que tout frappe, à qui rien n'échappe". Comme eux, il a des pressentiments, de ces "jugements subits auquels nous sommes entraînés par certaines circonstances très déliées" et qui sont "d'autant plus justes et plus prompts que nous avons plus de pénétration et d'expérience".

Leur erreur est de chercher une cause divine à ce don: "il leur semble que c'est une voix secrète qui parle au fond de leur coeur, et qui les avertit. Ils se croient inspirés, et ils le sont en effet, non par quelque puissance surnaturelle et divine, mais par une prudence particulière et extraordinaire" (VIII, 366). Ce qu'il appelle ici prudence n'est autre que cette faculté associative qu'il louait en 1753 chez les "manouvriers d'expériences" et il en parle presque dans les mêmes termes:

"Ils rapprochent les analogies les plus éloignées; ils voient des liaisons presque nécessaires où les autres sont loin d'avoir des conjectures".

Ils sont un peu ce qu'est Dieu dans l'harmonie universelle: "Dieu voit l'ordre de l'univers entier dans la plus petite molécule de la matière. La prudence de certains hommes privilégiés tient un peu de cet attribut de la Divinité".

On ne s'étonnera pas, dès lors, de le voir interrompre un peu plus loin une biographie de Paracelse par cette profession de foi:

"L'enthousiasme est le germe de toutes les grandes choses, bonnes ou mauvaises. Qui est-ce qui pratiquera la vertu au milieu des traverses qui l'attendent, sans enthousiasme? Qui est-ce qui se consacrera aux travaux continuels de l'étude, sans enthousiasme? Qui est-ce qui sacrifiera son repos, sa santé, son bonheur, sa vie, au progrès des sciences et des arts et à la recherche de la vérité, sans enthousiasme? Qui est-ce qui se ruinera, qui est-ce qui mourra pour son ami, pour ses enfants, pour son pays, sans enthousiasme?" (VIII, 368-369).

A bien des égards, Diderot s'identifie mentalement à Paracelse: lui aussi 
dépiste les secrets des métiers, lui aussi fréquente des gens de toute condition, lui aussi a plus appris de ceux que le monde appelle ignorants que de la science livresque, lui aussi n'est guère pieux; mais l'analogie s'arrête là, car Diderot ne voudrait être ni vaniteux, ni crapuleux, ni débauché, ni polygraphe.

Le génie étrange de ces théosophes, fait d'intuitions fulgurantes, continue cependant à le fasciner. Il le rapproche d'une folie qui tiendrait de l'hallucination et qui pourrait s'exercer dans le bien comme dans le mal. La longue digression (VIII, 390-391) sur la parenté entre Génie et Folie touche à une des questions majeures de l'esthétique de Diderot, dont on retrouvera d'ailleurs certains échos dans Le Neveu de Rameau.

"Je conjecture que ces hommes, d'un tempérament sombre et mélancolique, ne devaient cette pénétration extraordinaire et presque divine qu'on leur remarquait par intervalles, et qui les conduisait à des idées tantôt si folles, tantôt si sublimes, qu'à quelque dérangement périodique de la machine. Ils se croyaient alors inspirés, et ils étaient fous ... ils imaginaient que c'était la Divinité qui descendait, qui les visitait, qui les travaillait ... et ils donnaient des préceptes pour s'acheminer artificiellement à cet état d'orgasme et d'ivresse où ils se trouvaient au-dessus d'eux-mêmes et qu'ils regrettaient, semblables à ceux qui ont éprouvé l'enchantement et le délire délicieux que l'usage de l'opium porte dans l'imagination et dans les sens ... Ô que le génie et la folie se touchent de bien près! Ceux que le Ciel a signés en bien et en mal sont sujets plus ou moins à ces symptômes ... On les enferme et on les enchaîne, ou on leur élève des statues: ils prophétisent ou sur le trône, ou sur les théâtres, ou dans les chaires; ils tiennent l'attention des hommes suspendue; ils en sont écoutés, admirés, suivis ou insultés, bafoués, lapidés; leur sort ne dépend point d'eux, mais des circonstances dans lesquelles ils se montrent. Ce sont les temps d'ignorance et de grandes calamités qui les font naître: alors les hommes qui se croient poursuivis par la Divinité se rassemblent autour de ces espèces d'insensés, qui disposent d'eux. Ils ordonnent des sacrifices, et ils sont faits; des prières, et l'on prie; des jeûnes, et l'on jeûne; des meurtres, et l'on égorge; des chants d'allégresse et de joie, et l'on se couronne de fleurs, et l'on danse et l'on chante; des temples, et l'on en élève; les entreprises les plus désespérées, et elles réussissent; ils meurent, et ils sont adorés. Il faut ranger dans cette classe Pindare, Eschile, Mahomet, Shakespeare, Roger Bacon et Paracelse. Changez les instants, et celui qui fut poète eût été ou magicien, ou prophète, ou législateur. Ô hommes à qui la nature a donné cette grande et extraordinaire imagination, qui criez, qui subjuguez, que nous qualifions insensés ou sages, qui est-ce qui peut prédire votre destinée? vous naquîtes pour marcher entre les applaudissements de la terre ou l'ignominie, pour con- 
duire les peuples au bonheur ou au malheur, et laisser après vous le transport de la louange ou de l'exécration."

On aimerait connaître la date de rédaction de ce passage, publié en 1765, mais que Diderot a dû écrire pendant l'intervalle (1757-1765) qui sépare les deux séries de volumes de l'Encyclopédie. C'est précisément pendant cette même période que Diderot rédige les textes théoriques sur le théâtre dont bien des aspects semblent liés à l'article Théosophes par ces "rapports secrets" qu'évoque le début du Troisième Entretien sur le Fils Naturel.

Dorval est possédé par "les transports de son enthousiasme" (O.E., 135). Il est "sous le charme" du spectacle de la nature, ce "séjour sacré de l'enthousiasme". Il y cherche "un antre qui l'inspire". bientôt le spectacle de l'eau soulève son âme et enflamme son imagination. "Le moment de l'enthousiasme" s'annonce "par un frémissement qui part de sa poitrine et qui passe, d'une manière délicieuse et rapide, jusqu'aux extrémités de son corps" pour se muer bientôt en "une chaleur forte et permanente qui l'embrase, qui le fait haleter, qui le consume, qui le tue". Dorval monologue en état de délire et il en sort comme d'un profond sommeil (O.E., 98).

Le poète est tenu pour une sorte de mystique, accédant à un état second où il vaticine, et se confond ainsi avec le prophète ou avec le mage. Il est, ou se croit, possédé par une force mystérieuse qui s'est emparée de lui. L'art, l'amour et la religion sont les domaines privilégiés de ces états de transes:

“Les poètes, les acteurs, les musiciens, les peintres, les chanteurs de premier ordre, les grands danseurs, les amants tendres, les vrais dévots, toute cette troupe enthousiaste et passionnée sent vivement, et réfléchit peu. Ce n'est pas le précepte; c'est autre chose de plus immédiat, de plus intime, de plus obscur et de plus certain qui les guide et qui les éclaire" (O.E., 104).

Sans le dire, Diderot renoue ici avec la théorie néo-platonicienne du poète-vates, possédé par son "démon", éclairé par une lumière intérieure qui exclut le recours à la raison raisonnante.

Le refus de la raison explique que ces inspirés émergent toujours aux "temps d'ignorance et de grandes calamités", donc à des périodes où la pensée analytique capitule devant l'irrationnel, qui vient combler le vide laissé dans les esprits. Il en est aussi question dans le Second Entretien, par la voix de Dorval: "alors les hommes se meuvent dans les ténèbres. Ce sont les temps de l'ignorance et du crime, du fanatisme et des conquêtes" (O.E., 128). Mais c'est surtout dans le fameux pas- 
sage du chapitre XVIII, Des Moeurs, dans le traité De la poésie dramatique (1758), que le rapprochement entre poésie et barbarie est poussé jusqu'à ses limites. On connaît ce morceau lyrique, trop souvent cité comme le credo poétique définitif de Diderot:

"La poésie veut quelque chose d'énorme, de barbare et de sauvage. C'est lorsque la fureur de la guerre civile ou du fanatisme arme les hommes de poignards, et que le sang coule à grands flots sur la terre, que le laurier $\mathrm{d}^{\prime}$ Apollon s'agite et verdit ... Quand verra-t-on naître des poètes? Ce sera après les temps de désastres et de grands malheurs ... (O.E., 261-262).

Nous avons étudié ailleurs ${ }^{9}$ le contexte de cette surprenante vaticination. Inquiet du déclin de la poésie, saturé par instants de culture et de rationalité, grand amateur $\mathrm{d}^{\prime}$ Homère, lecteur passionné qui vient de découvrir dans Helvétius l'atroce grandeur de l'Edda, Diderot rêve à haute voix de grandeur sauvage et de barbarie héroïque. Mais on s'égarerait en prenant ces délires inspirés pour un programme d'avenir. Diderot aime à s'en délecter rétrospectivement, par la lecture ou par le biais de la peinture d'histoire. Ces bouffées primitivistes lui servent d'antidote à un excès de sociabilité et de délicatesse, à des moeurs modernes "faibles, petites et maniérées". L'artiste a le droit d'y rêver, et même d'y prendre un bain de sauvagerie rafraîchissante. Le philosophe ne saurait les proposer sérieusement en modèle. En somme, il arrive à Diderot de se prendre au piège de son propre lyrisme.

Dans la mesure cependant où l'article Théosophes associe création poétique (Pindare, Eschyle, Shakespeare), théosophie (Paracelse), religion (Mahomet), et action politique (législateur, sur le trône), Diderot est conscient de l'ambiguité de la notion même d'enthousiasme, génératrice de grandeur, de beauté sublime, mais aussi d'horreur et de tyrannie.

Aussi, à la réflexion, clôture-t-il son article assez dithyrambique par une importante restriction, qui tourne aussitôt à la condamnation. L'enthousiasme, lorsqu'il se transfère du poétique au politique et surtout au domaine religieux, manifeste rapidement ses virtualités maléfiques. Les théosophes modernes sont des esprits dangereux. Ils déshonorent la Révélation, ils rétrécissent l'empire de la raison; ils taxent d'hérésie toute idée nouvelle; ils réduiraient volontiers toute connaissance à celle de la religion et toute lecture à celle de la Bible; ils "ont pris en aversion la philosophie et les philosophes (et) réussiraient à éteindre par$\mathrm{mi}$ nous l'esprit de découvertes et de recherches, et à nous replonger dans la barbarie, si le gouvernement les appuyait, comme ils le demandent" (VIII, 392-393). 
Soudain dégrisé de ses ferveurs lyriques, Diderot mesure le grave danger que représente, pour ses amis "philosophes" et pour la cause qu'ils servent, un concept si aisément détourné vers le fanatisme et vers l'aliénation religieuse. L'ambiguïté de l'enthousiasme lui apparaît soudain clairement, et il en réprouve la version moderne, dégénérée et menaçante. Pour Pindare, pour un Shakespeare, que d'Abraham Chaumeix, que d'Omer Joly de Fleury!

Mieux que tout autre texte, l'article Théosophes éclaire le double mouvement de la pensée de Diderot devant les séductions de l'enthousiasme (ou du "feu intérieur") et devant les perversions qu'il est capable d'engendrer. L'esthète et le philosophe militant se contredisent parfois en lui, même si, en profondeur, l'esthète ne cesse d'éclairer la démarche du penseur, toujours en quête d'analogies, de liaisons cachées. Esthétiquement, l'enthousiasme est source de grandeur, mais cette grandeur peut s'exercer dans le mal comme dans le bien (le neveu de Rameau le soulignera complaisamment). Diderot met son lecteur en garde contre ses propres effusions et il ne retrouve son sang-froid philosophique que pour réprouver les aspects religieux d'un phénomène dont les autres facettes le fascinent pourtant.

Au cours des années ultérieures, la réflexion philosophique et scientifique de Diderot va s'engager dans une voie différente. L'étude du cerveau et des phénomènes nerveux, résumée dans l'image de la toile d'araignée que le Dr Bordeu évoque dans Le rêve de d'Alembert, l'importance croissante accordée au sensorium commune par rapport aux sensations périphériques, une certaine autocritique aussi, vont amener Diderot à revoir en termes nouveaux l'opposition de l'enthousiaste et de l'esprit froid. Conçu en parallèle avec Le Rêve, et dans son prolongement, le Paradoxe sur le Comédien fixe très clairement ce qu'est devenue la position de Diderot à la fin de 1769.

Le Paradoxe a sans aucun doute un caractère provocant et radical, mais on aurait tort de n'y voir qu'une foucade, un caprice, un désir de surprendre et de choquer. Lui-même écrivait à Grimm, le 14 novembre 1769: "Avec un peu de soin, je n'aurais peut-être jamais rien écrit où il y eût plus de finesse et de vue". Le ton péremptoire du Premier n'est si tranchant que parce que Diderot s'y réfute lui-même et qu'il souligne délibérément le virage pris par sa pensée en matière esthétique.

Il admet l'importance du don naturel, mais il le juge insuffisant: l'étude des grands modèles, la connaissance du coeur humain, l'expérience, l'usage du monde, et surtout un travail assidu doivent le perfectionner. Pour lui, la qualité première de l'acteur n'est pas la sensibilité (comme il l'affirmait en 1758), mais le jugement: 
"Il me faut dans cet homme un spectateur froid et tranquille; j'en exige, par conséquent, de la pénétration et nulle sensibilité" (O.E., 306).

Ce n'est pas que Diderot ignore ou conteste le rôle de l'inspiration, mais il ne la situe plus au climax de l'émotion, où elle se confondrait avec l'impact de la sensibilité. Ce qu'il appelle "le moment sublime" est postérieur à la phase émotive, et il étend sa constatation à l'artiste en général, qui n'est grand qu'au moment où il parvient à se dédoubler.

"Et pourquoi l'acteur différerait-il du poète, du peintre, de l'orateur, du musicien? Ce n'est pas dans la fureur du premier jet que les traits caractéristiques se présentent, c'est dans des moments tout à fait inattendus ... c'est au sang-froid à tempérer le délire de l'enthousiasme" (O.E., 309).

On ne saurait être plus clair. Pourtant le Premier reviendra à la charge, longuement, comme s'il pressentait la difficulté de convaincre le lecteur et de renverser les idées reçues.

- “La sensibilité n'est guère la qualité d'un grand génie ... Ce n'est pas son coeur, c'est sa tête qui fait tout" (O.E., 310),

ou encore

- “La sensibilité n'est jamais sans faiblesse d'organisation [c'est la thèse de Bordeu dans le Rêve] ... dans la grande comédie du monde, toutes les âmes chaudes occupent le théâtre; tous les hommes de génie sont au parterre. Les premiers s'appellent des fous; les seconds, qui s'occupent à copier leurs folies, s'appellent des sages" (O.E., 311).

Au fur et à mesure que le dialogue progresse, le Premier s'identifie de plus en plus avec Diderot et la part d'autocritique se fait plus évidente, mais aussi d'autant plus pertinente pour la justification de sa thèse:

“lorsque j'ai prononcé que la sensibilité était la caractéristique de la bonté de l'âme et de la médiocrité du génie, j'ai fait un aveu qui n'est pas trop ordinaire, car si Nature a pétri une âme sensible, c'est la mienne" (O.E., 362).

Le jeu du grand acteur, de même que le processus de création chez l'artiste, sera donc, en définitive, "l'ouvrage d'une tête froide, d'un profond jugement, d'un goût exquis, d'une étude pénible, d'une longue expérience et d'une ténacité de mémoire peu commune" (O.E., 372). 
Diderot aurait-il, une fois pour toutes, condamné cet enthousiasme qu'il avait si chaleureusement loué à tant d'occasions? La réponse n'est pas aussi simple, car la thèse du Paradoxe concerne le moment de la représentation pour l'acteur, et celui la composition pour l'artiste. Entraîné par sa propre chaleur argumentative, Diderot en arrive à durcir, par moments, une réalité infiniment complexe et, à s'exprimer en termes trop absolus ("de la pénétration et nulle sensibilité"). Ailleurs, il semble distinguer entre les phases du processus créateur, où un premier mouvement d'enthousiasme sera ensuite tempéré par le sang-froid et transposé en "modèle idéal". Surtout, l'enthousiasme lui est suspect parce qu'il tient du délire, qu'il est incompatible avec les exigences formelles de l'art, et enfin parce qu'il est aisé à contrefaire dans ses manifestations extérieures.

Après les apologies lyriques de 1758 , le balancier est allé cette fois à l'extrême opposé. Cependant, on le sait par Le Rêve de d'Alembert, “notre véritable sentiment n'est pas celui dans lequel nous n'avons jamais vacillé, mais celui auquel nous sommes le plus habituellement revenus" $(O . P h ., 283)$. Or Diderot reviendra assez vite à une conception plus positive de l'enthousiasme, où l'élan poétique et une certaine ferveur retrouvent une adhésion momentanément sacrifiée à un "beau paradoxe".

Dans ce qu'il est convenu d'appeler la Réfutation d'Helvétius (1773-1774), il critique l'utilitarisme du livre De l'Homme en tirant argument de l'esprit de sacrifice désintéressé dont font preuve tant de philosophes:

"Comment résoudrez-vous en dernière analyse à des plaisirs sensuels, sans un pitoyable abus de mots, ce généreux enthousiasme qui les expose à la perte de leur liberté, de leur fortune, de leur honneur et de leur vie?" (O.Ph., 573).

Plus loin, parlant du poète, il l'oppose à "l'homme froid" et à "l'esprit réglé":

"Je voudrais bien savoir comment l'intérêt, l'éducation, le hasard, donnent de la chaleur à l'homme froid, de la verve à l'esprit réglé, de l'imagination à celui qui n'en a point ... Plus j'y rêve, plus le paradoxe de l'auteur me confond. Si cet artiste n'est pas né ivre, la meilleure instruction ne lui apprendra jamais qu'à contrefaire plus ou moins maussadement l'ivresse. De là tant de plats imitateurs de Pindare et de tous les auteurs originaux" (O.Ph., 577 ).

On se souvient de la place qu'il accordait à Pindare dans l'article Théosophes, à côté des "inspirés" et des "insensés". Or c'est la même conception de la poésie qu'il va défendre contre Helvétius un peu plus loin: 
"La poésie suppose une exaltation de tête qui tient presque à l'inspiration divine. Il vient au poète des idées profondes dont il ignore et le principe et les suites. Fruits d'une longue méditation dans le philosophe, il en est étonné, il s'écrie: 'Qui est-ce qui a inspiré tant de sagesse à cette espèce de fou-là?' " $(O . P h .$, $591)$.

Si le véritable sentiment est celui auquel on revient le plus habituellement (pour employer sa propre formule), Diderot est bien plus l'homme de l'enthousiasme (sauf au sens mystico-religieux) que celui de la tête froide. S'il lui est arrivé de parler éloquemment en faveur de la seconde, c'est pour se purger de ses propres défauts et pour souligner la part de concentration et de travail qui entre dans toute création artistique. En profondeur, Diderot reste l'homme de l'élan, de la chaleur, de l'exaltation de tête, comme il dit ${ }^{10}$. Il se sent proche de cette troupe "enthousiaste et passionnée" où se rejoignent poètes, musiciens et danseurs.

Prenons-y garde d'ailleurs: s'il fait l'éloge du spectateur "froid et tranquille", c'est surtout en raison de son pouvoir de concentration et du contrôle qu'il est capable d'exercer sur lui-même (qualité qui lui fait complètement défaut et qu'il n'en admire que plus vivement). Il le voit comme un homme supérieur, comme un génie doué d'une exceptionnelle pénétration d'esprit. Jamais il ne le confond avec l'esprit rassis, pragmatique et sec de ceux qu'il appelle les "méthodistes", pour lesquels il n'éprouve invariablement qu'un parfait mépris.

La fable du coucou et du rossignol illustre bien ses préférences. Le coucou sait peu de choses, mais elles ont du poids, de l'ordre, et on les retient. Le rossignol, toujours nouveau, se joue des règles et enchante les forêts. Le coucou possède l'artifice et la méthode. Le rossignol se lance dans les modulations les plus hardies, suit les chants les plus neufs et les plus recherchés; il est emporté par l'enthousiasme; son chant est successivement doux, léger, brillant, pathétique, mais il n'est pas fait pour tout le monde. Aussi le jugement de l'âne, qui n'y a rien entendu, sera-t-il négatif:

"cela me paraît bizarre, brouillé, décousu. Vous êtes peut-être plus savant que votre rival, mais il est plus méthodique que vous, et je suis, moi, pour la méthode" (à Sophie Volland, le 20 octobre 1760, Roth III, 167-169).

Si la méthode plaît aux ânes, Diderot, en tout cas, l'a en horreur: "Je ne saurais m'accommoder de ces gens stricts. Il ressemblent à ces écureuils du quai de la Ferraille qui font sans cesse tourner leur cage, les plus misérables créatures qu'il y ait" (à Sophie Volland, 5 août 1762; 
Roth IV, 88). Ecrivant en juin 1770 à l'abbé Galiani, il constate que depuis son départ pour Naples, on n'entend plus au Grandval que "les criailleries aigres et sèches" de l'abbé (Morellet) et "la grosse et lourde raison de notre ami Marmontel" (Roth, X, 67).

Dans le Salon de 1767, parlant du critique, il le montre arrachant les plumes à Pégase, et ajoute: “C'est une bête de somme, la monture de l'abbé Morellet, prototype de la méthode la discipline militaire naît quand il n'y a plus de généraux; la méthode, quand il n'y a plus de génie".

Diderot est douloureusement sensible, lui qui est à la fois poète et philosophe, à la décadence de la poésie entraînée par les progrès de l'esprit philosophique, qui requiert le jugement au détriment de l'imagination.

Dans le même Salon, il constate que "l'esprit philosophique amène le style sentencieux et $\mathrm{sec}^{\prime \prime}$, et il le déplore en constatant le déclin de l'ode:

"Le philosophe raisonne, l'enthousiaste sent. Le philosophe est sobre, l'enthousiaste est ivre ... Allez à cinq heures sous les arbres des Tuileries. Là, vous trouverez de froids discoureurs placés parallèlement les uns à côté des autres; mesurant d'un pas égal des allées parallèles; aussi compassés dans leurs propos que dans leur allure, étrangers aux tourments de l'âme d'un poète, qu'ils n'éprouvèrent jamais. Et vous entendrez le dithyrambe de Pindare traité d'extravagance; et cet aigle endormi sous le sceptre de Jupiter, qui se balance sur ses pieds, et dont les plumes frissonnent aux accents de l'harmonie, mis au rang des images puériles. Quand voit-on naître les critiques et les grammairiens? Tout juste après le siècle du génie et des productions divines. Ce siècle s'éclipse pour ne plus reparaître ... Le génie crée les beautés; le critique remarque les défauts. Il faut de l'imagination pour l'un, du jugement pour l'autre".

Mais si la sympathie de Diderot va aux enthousiastes, aux poètes, aux natures entières et ferventes, il sait aussi d'expérience que ces êtres d'exception sont voués au malheur dans notre ordre social:

"Il ne faut point faire de poésie dans la vie. Les héros, les amants romanesques, les grands patriotes, les magistrats inflexibles, les apôtres de religion, les philosophes à toute outrance, tous ces rares et divins insensés font de la poésie dans la vie; de là leur malheur".

Le monde appartient aux médiocres, aux "méthodistes". L'éloge sarcastique de M. Baliveau rejoint celui de Briasson dans Le Neveu de 
Rameau. Autre chose est de savoir si Diderot souhaite personnellement ordonner sa vie sur un tel modèle. Il a beau faire l'éloge du juste milieu; la question qui se pose à lui est de savoir "pourquoi personne ne voudrait perdre de sa sensibilité et devenir médiocre".

Lui-même n'y a jamais songé, quitte à passer pour "un énergumène" (Corr., X, 67) aux yeux des froids raisonneurs.

La philosophie, à laquelle il a voué sa vie, ne saurait la remplir intégralement. Elle est "l'opium des passions, la vieillesse d'un moment" (Corr. XVI, 66). Diderot sait que son siècle est celui de la philosophie, et il s'en félicite à maintes reprises, mais il voit dans cette sagesse tranquille l'effet de l'âge et du vieillissement. Ses bouffées primitivistes, ses élans d'enthousiasme, sont autant d'expressions de sa nostalgie d'une époque plus sauvage et plus poétique. Toute poésie, écrit-il dans le Salon de 1767, comporte une part de mensonge; la philosophie le dénonce à bon droit, mais elle évacue la poésie en même temps que ses écarts. A l'inverse de Voltaire, Diderot ne croit pas à "l'enthousiasme raisonnable". Celui que ses contemporains appelaient "le philosophe" ne sortira jamais de ce dilemme qui le déchire au plus profond de son être. Il sait la nécessité du jugement et de l'esprit critique, serviteurs de la vérité, mais il en décèle les limites et le côté desséchant. Il connaît les dangers de l'enthousiasme, sa proximité des excès mystiques, du fanatisme et des formes inquiétantes de l'aliénation, mais il y voit aussi la source de l'authentique poésie et de la vraie grandeur.

Il arrive à Diderot de se rêver observateur froid et tranquille; il lui arrive même de prêcher pour le bonheur dans la médiocrité. Pourtant, dans le secret de sa conscience, il reste le frère en esprit de ces inspirés, de ces insensés que sont les poètes et les théosophes. Si Diderot n'a pas écrit de système, si son discours philosophique s'écarte de celui de Condillac ou de son ami d'Holbach, si son dialogue reste ouvert et sa pensée questionneuse, si même ses articles encyclopédiques portent l'empreinte de sa subjectivité passionnée, c'est peut-être parce qu'il a tenté ce qui, dans son esprit, devait être le paradoxe suprême: la conciliation de la poésie et de la pénétration, de l'esprit froid et de l'enthousiasme.

ROLAND MORTIER

Université libre de Bruxelles 


\section{NOTES}

1. Voir à ce sujet les excellentes études de Werner Krauss, Über frz. "enthousiasme" im 78. Jhdt., dans "Wiss. Zeitung Un. Halle", XIX, 1970, H. 3/4, p. 91-100, et de Fritz Schalk, Zur Geschichte von "Enthousiasme", dans Romanische Forschungen, 1975, t. 87/2, p. 191-225, ainsi que l'ouvrage de S. Tucker, Enthusiasm. A study in semantic change, London, 1972, 224 p., et l'excellent article Enthousiasme des Schlüsselbegriffe des kunsttheoretischen Denkens in Frankreich von der Spätklassik bis zum Ende der Aufklärung (Düsseldorf, Schwann, 1972), de P.E. Knabe.

2. Robespierre (Discours sur la guerre, 1792): "la France entière, animée du saint enthousiasme de la liberté". Madame de Staël lui consacre les chapitres X, XI et XII de la IVe Partie de De l'Allemagne et s'insurge contre la confusion de l'enthousiasme avec le fanatisme. Pour elle, "l'enthousiasme se rallie à l'harmonie universelle: c'est l'amour du beau, l'élévation de l'âme, la jouissance du dévouement, réunis dans un même sentiment qui a de la grandeur et du calme ... l'enthousiasme est tout pour les nations littéraires ... l'enthousiasme seul est inaltérable ...". Vigny (Journal du poète): "Ce n'est pas pour rien qu'enthousiasme veut dire Dieu en nous". Baudelaire (Paradis artificiels): "L'enthousiasme et la volonté suffisent pour élever l'homme à une existence supranaturelle". G. Sand (Correspondance, 1866): "La foi est une surexcitation, un enthousiasme, un état de grandeur intellectuelle". Par contre, Valéry (Variété, I, 1924): "L'enthousiasme n'est pas un état d'âme d'écrivain". Fort curieusement, Chateaubriand maintient le sens dépréciatif du XVIIIe siècle. Il dit, dans l'Epilogue $\mathrm{d}^{\prime}$ Atala, avoir voulu montrer "les dangers de l'ignorance et de l'enthousiasme religieux, opposés aux lumières, à la charité et au véritable esprit de l'Evangile".

3. Encore que Voltaire, dans ses annotations, considère que dès le moment où ces passions s'équilibrent dans une juste harmonie (cf. Pensée IV), "ce balancement est sagesse; il exclut l'enthousiasme". Il est douteux qu'une telle conclusion aurait pu être acceptée par Diderot à cette date. Voltaire fera plus tard l'apologie de "l'enthousiasme raisonnable" (Dict. phil., article Enthousiasme, qu'il dit être "le partage des grands poètes". A propos du même article de l'Encyclopédie, il avait écrit à d'Alembert (le 13 novembre 1756): "on n'a que faire d'un si long discours pour savoir que l'enthousiasme doit être gouverné par la raison".

4. Dans leur introduction aux Pensées philosophiques (éd. Hermann, t. II, 1975, p. 5), Robert Niklaus et Yvon Belaval parlent, fort justement, d'un "caractère d'ambiguité que certains flottements ne font que renforcer".

5. Et même, plus tard encore, dans sa Réfutation d'Helvétius: "fait-on des expériences au hasard? L'expérience n'est-elle pas souvent précédée d'une supposition, d'une analogie ..." (Oeuvres philosophiques, éd. Vernière, p. 598).

6. Ce phénomène, lié selon lui à une intense concentration de l'esprit, l'a beaucoup frappé (cf. le prêtre de Calame, dans la Pensée philosophique LI, et le curé Moni de Langres dans Le rêve de d'Alembert, Oeuvres philosophiques, éd. Vernière, p. 351). 
7. C'est pourqui il souhaite (art. Encyclopédie) que l'éditeur d'un dictionnaire encyclopédique ne soit "jamais enthousiaste, à moins que ce ne fût de la vérité, de la vertu, et de l'humanité" (éd. Hermann, VII, 261).

8. Voir le Cahier XIII de l'A.I.E.F. (1961).

9. Voir Diderot au carrefour de la poésie et de la philosophie, dans "Revue des Sciences humaines", octobre-décembre 1963, pp. 485-501.

10. Voici comment Julie de Lespinasse le voit en juin 1773: "C'est un homme extraordinaire, il n'est pas à sa place dans la société. Il devrait être chef de secte, un philosophe instruisant, enseignant la jeunesse. Il me plaît fort, mais rien de toute sa manière ne vient à mon âme: sa sensibilité est à fleur de peau; il ne va pas plus loin que l'émotion" (Corr. XIII, 17). Pour Mme Geoffrin, "il est toujours comme un homme qui rêve, et qui croit réel tout ce qu'il a rêvé" (Corr., XIII, 242). 